\title{
Harish-Chandra Isomorphisms for Quantum Algebras
}

\section{Toshiyuki Tanisaki}

Department of Mathematics, College of General Education, Osaka University, Toyonaka 560, Japan

\begin{abstract}
The center of the quantum algebra is studied. Especially an analogue of the Harish-Chandra isomorphism is established.
\end{abstract}

\section{Introduction}

In the study of the quantum Yang-Baxter equation, Drinfel'd [3] and Jimbo [6] found a certain Hopf algebra, which is a quantization of the enveloping algebra of a symmetrizable Kac-Moody Lie algebra (The $\mathfrak{s l}_{2}$ case is due to KulishReshetikhin and Sklyanin). The purpose of this paper is to investigate the structure of the center of this quantum algebra associated to a finite dimensional semisimple Lie algebra. Our main result is Theorem 2 below giving an analogue of the Harish-Chandra isomorphism ([5]).

Let $A=\left(a_{i j}\right)_{1 \leqq i, j \leqq l}$ be a symmetrizable generalized Cartan matrix. This means that $A$ is a matrix of integers such that $a_{i i}=2, a_{i j} \leqq 0$ for $i \neq j$ and there exist positive integers $d_{1}, \ldots, d_{l}$ satisfying $d_{i} a_{i j}=d_{j} a_{j i}$. We fix such $d_{1}, \ldots, d_{l}$. Let $k$ be a field of characteristic zero. Choose a finite-dimensional $k$-vector space $t_{0}$ and elements $\alpha_{1}, \ldots, \alpha_{l} \in \mathfrak{t}_{0}^{*}, t_{1}, \ldots, t_{l} \in \mathrm{t}_{0}$ satisfying the following conditions:

(a) $\left\{\alpha_{1}, \ldots, \alpha_{l}\right\}$ is linearly independent,

(b) $\left\{t_{1}, \ldots, t_{l}\right\}$ is linearly independent,

(c) $\alpha_{i}\left(t_{j}\right)=d_{i} a_{i j}(i, j=1, \ldots, l)$.

The Kac-Moody Lie algebra $g$ (see [8]) associated to $A$ is the Lie algebra over $k$, generated by the $k$-vector space $\mathrm{t}_{0}$ and the elements $e_{1}, \ldots, e_{l}, f_{1}, \ldots, f_{l}$ with the following fundamental relations:

$$
\begin{gathered}
{\left[t, t^{\prime}\right]=0 \quad\left(t, t^{\prime} \in \mathrm{t}_{0}\right),} \\
{\left[t, e_{i}\right]=\alpha_{i}(t) e_{i} \quad\left(t \in \mathrm{t}_{0}, i=1, \ldots, l\right),} \\
{\left[t, f_{i}\right]=-\alpha_{i}(t) f_{i} \quad\left(t \in \mathrm{t}_{0}, i=1, \ldots, l\right),} \\
{\left[e_{i}, f_{j}\right]=\delta_{i, j} t_{i} / d_{i} \quad(i, j=1, \ldots, l),}
\end{gathered}
$$




$$
\begin{array}{ll}
\left(\operatorname{ad}\left(e_{i}\right)\right)^{1-a_{i j}}\left(e_{j}\right)=0 & (i \neq j) \\
\left(\operatorname{ad}\left(f_{i}\right)\right)^{1-a_{i j}}\left(f_{j}\right)=0 & (i \neq j) .
\end{array}
$$

We introduce the following $q$-analogue of the binomial coefficient $\left(\begin{array}{l}n \\ m\end{array}\right)$ for non-negative integers $n, m$ with $n \geqq m$ :

$$
\left[\begin{array}{l}
n \\
m
\end{array}\right]_{q}=\frac{\prod_{r=1}^{n}\left(q^{r}-q^{-r}\right)}{\prod_{r=1}^{m}\left(q^{r}-q^{-r}\right) \prod_{r=1}^{n-m}\left(q^{r}-q^{-r}\right)}
$$

The quantum algebra $U_{h}(\mathrm{~g})$ is the algebra over the formal power series ring $R=k[[\hbar]]$, which is $\hbar$-adically generated by the $R$-module $\mathrm{t}=R \otimes_{k} \mathrm{t}_{0}$ and the elements $e_{1}, \ldots, e_{l}, f_{1}, \ldots, f_{l}$ with the following fundamental relations:

$$
\begin{aligned}
& t t^{\prime}-t^{\prime} t=0\left(t, t^{\prime} \in \mathrm{t}\right), \\
& t e_{i}-e_{i} t-\alpha_{i}(t) e_{i}=0(t \in \mathrm{t}, i=1, \ldots, l), \\
& t f_{i}-f_{i} t+\alpha_{i}(t) f_{i}=0(t \in \mathrm{t}, i=1, \ldots, l), \\
& e_{i} f_{j}-f_{j} e_{i}-\delta_{i, j} \frac{\sinh \left(\hbar t_{i} / 2\right)}{\sinh \left(\hbar d_{i} / 2\right)}=0 \quad(i, j=1, \ldots, l), \\
& \sum_{m=0}^{1-a_{i j}}(-1)^{m}\left[\begin{array}{c}
1-a_{i j} \\
m
\end{array}\right]_{q_{i}} e_{i}^{1-a_{i j}-m} e_{j} e_{i}^{m}=0(i \neq j), \\
& \sum_{m=0}^{1-a_{i j}}(-1)^{m}\left[\begin{array}{c}
1-a_{i j} \\
m
\end{array}\right]_{q_{i}} f_{i}^{1-a_{i j}-m} f_{j} f_{i}^{m}=0(i \neq j),
\end{aligned}
$$

where $q_{i}=\exp \left(\hbar \alpha_{i}\left(t_{i}\right) / 4\right) \in R^{*}$ for $i=1, \ldots, l$. Let us be more precise. Let $U$ be the tensor algebra of the free $R$-module $\mathrm{t} \oplus\left(\bigoplus_{i=1}^{l} R e_{i}\right) \oplus\left(\bigoplus_{i=1}^{l} R f_{i}\right)$. We denote by $\hat{U}$ the completion of $U$ with respect to the $\hbar$-adic topology (see Sect. 2.1 below). Hence we have $\hat{U}=\lim \left(U / \hbar^{n} U\right)$ and $\hat{U}$ has a natural $R$-algebra structure. Let $I$ be the two-sided ideal of $\hat{U}$ generated by the left-hand sides of $(1.8), \ldots,(1.13)$ and let $\bar{I}$ be its closure with respect to the $\hbar$-adic topology. Then $\bar{I}$ is also a two-sided ideal of $\hat{U}$, and we define $U_{\hbar}(\mathfrak{g})$ to be the quotient algebra $\hat{U} / \bar{I}$. Apparently $U_{\hbar}(\mathfrak{g}) / \hbar U_{h}(\mathfrak{g})$ is naturally isomorphic to the enveloping algebra $U(\mathfrak{g})$ of $\mathfrak{g}$.

Let $N^{+}$(respectively $N^{-}$, respectively $T$ ) be the subalgebra of $U_{h}(\mathrm{~g})$ generated by $e_{1}, \ldots, e_{l}$ (respectively $f_{1}, \ldots, f_{l}$, respectively $\mathrm{t}$ ), and let $U_{h}^{f}(\mathrm{~g})$ be the subalgebra generated by $N^{+}, N^{-}, \bar{T}$, where barring denotes the $\hbar$-adic closure. We denote by $N$ the $R$-algebra with 1 which is given by the presentation with generators $v_{1}, \ldots, v_{l}$ and relations:

$$
\sum_{m=0}^{1-a_{i_{j}}}(-1)^{m}\left[\begin{array}{c}
1-a_{i j} \\
m
\end{array}\right]_{q_{i}} v_{i}^{1-a_{i j}-m} v_{j} v_{i}^{m}=0 \quad(i \neq j)
$$


Theorem 1. (i) $N$ is a free $R$-module and we have isomorphisms of $R$-algebras:

$$
N^{+} \simeq N \simeq N^{-} \quad\left(e_{i} \leftrightarrow v_{i} \leftrightarrow f_{i}\right)
$$

(ii) $T$ is naturally isomorphic to the symmetric algebra $S(\mathrm{t})$ of the free $R$-module $\mathrm{t}$, and the inclusion $T \subset \bar{T}$ is the $\hbar$-adic completion.

(iii) We have an isomorphism of $R$-modules:

$$
U_{h}^{f}(\mathfrak{g}) \simeq N^{-} \otimes \bar{T} \otimes N^{+} \quad(u v w \leftrightarrow u \otimes v \otimes w) .
$$

(iv) The inclusion $U_{\hbar}^{f}(\mathrm{~g}) \subset U_{\hbar}(\mathrm{g})$ is the $\hbar$-adic completion.

This theorem may be well-known to the experts (see [3]); however, we will give its proof since it seems that it does not exist in the literature and since it will be used in the proof of our main result. The proof of the freeness of $N$ is based on the character formula of integrable highest weight modules (Lusztig [9]), and the other statements are proved using the arguments of Yamane [13].

The topological Hopf algebra structure on $U_{\hbar}(\mathfrak{g})$ defined by the following is one of the ingredients of the proof of our main result (see [3], [6]):

$$
\begin{gathered}
\Delta(t)=t \otimes 1+1 \otimes t \quad(t \in \mathfrak{t}), \\
\Delta\left(e_{i}\right)=e_{i} \otimes \exp \left(-\frac{\hbar t_{i}}{4}\right)+\exp \left(\frac{\hbar t_{i}}{4}\right) \otimes e_{i} \quad(i=1, \ldots, l), \\
\Delta\left(f_{i}\right)=f_{i} \otimes \exp \left(-\frac{\hbar t_{i}}{4}\right)+\exp \left(\frac{\hbar t_{i}}{4}\right) \otimes f_{i} \quad(i=1, \ldots, l), \\
\varepsilon(\mathrm{t})=0 \quad \varepsilon\left(e_{i}\right)=\varepsilon\left(f_{i}\right)=0 \quad(i=1, \ldots, l) \\
S(t)=-t \quad(t \in \mathfrak{t}), \\
S\left(e_{i}\right)=-q_{i}^{-1} e_{i} \quad(i=1, \ldots, l), \\
S\left(f_{i}\right)=-q_{i} f_{i} \quad(i=1, \ldots, l) .
\end{gathered}
$$

Here $\Delta, \varepsilon, S$ are the coproduct, the counit and the antipode, respectively. Note that the definition of the topological Hopf algebra structure on an $R$-algebra $H$ is given by replacing $H \otimes H, H \otimes H \otimes H$ in the definition of the Hopf algebra (see [1]) with their $\hbar$-adic completions.

In the rest of this section we assume that $A$ is a Cartan matrix of finite type (see [8, Chap. 4]) and $t_{0}$ is spanned by $t_{1}, \ldots, t_{l}$. Therefore $g$ is a finite-dimensional semisimple Lie algebra and $t_{0}$ is identified with a split Cartan subalgebra of $g$. Let $W$ be the Weyl group, that is, the subgroup of the automorphism group of the $k$-vector space $\mathrm{t}_{0}$ generated by the transformations $s_{1}, \ldots, s_{l}$ given by:

$$
s_{i}\left(t_{j}\right)=t_{j}-a_{i j} t_{i} \text {. }
$$

Since the action of $W$ on $\mathrm{t}_{0}$ is naturally extended to an $R$-linear action on $\mathrm{t}=R \otimes \mathrm{t}_{0}$, the group $W$ acts on $T$ and $\bar{T}$ as algebra automorphisms. Define $\rho \in \mathrm{t}_{0}^{*} \subset \operatorname{Hom}_{R}(\mathrm{t}, R)$ by $\rho\left(t_{i}\right)=\alpha_{i}\left(t_{i}\right) / 2=d_{i}$ for $i=1, \ldots, l$.

We define an analogue of the Harish-Chandra homomorphism as follows. Let $\varepsilon^{ \pm}: N^{ \pm} \rightarrow R$ be the algebra homomorphisms defined by $\varepsilon^{+}\left(e_{i}\right)=0, \varepsilon^{-}\left(f_{i}\right)=0$ for 
$i=1, \ldots, l$ and let $\beta$ be the unique algebra automorphism of $\overline{\bar{T}}$ satisfying $\beta(t)=t-\rho(t)$ for $t \in \mathrm{t}$. Identifying $U_{h}^{f}(\mathrm{~g})$ with $N^{-} \otimes \bar{T} \otimes N^{+}$by Theorem 1 (iii), we define $\hat{\delta}: U_{h}^{f}(\mathrm{~g}) \rightarrow \bar{T}$ by $\hat{\delta}=\varepsilon^{-} \otimes \beta \otimes \varepsilon^{+}$. By virtue of Theorem 1 (iv) $\hat{\delta}$ is uniquely extended to an $R$-module homomorphism $\hat{\delta}: U_{h}(\mathrm{~g}) \rightarrow \bar{T}$. Let $z\left(U_{h}(\mathrm{~g})\right)$ be the center of $U_{h}(\mathfrak{g})$ and let $\delta: z\left(U_{h}(\mathfrak{g})\right) \rightarrow \bar{T}$ be the restriction of $\hat{\delta}$.

Theorem 2. $\delta$ is an injective algebra homomorphism and its image coincides with $\bar{T}^{W}=\{z \in \bar{T} \mid w(z)=z$ for all $w \in W\}$.

The organization of this paper is as follows. In Sect. 2 we will give a proof of Theorem 1 except for the freeness of $N$. In Sect. 3 several basic facts concerning highest weight modules are stated and a proof of the freeness is given. Section 4 is devoted to the proof of Theorem 2 .

\section{Structure of $U_{\hbar}(g)$}

2.1. $\hbar$-Adic Topologies. We recall basic facts concerning the topologies of modules defined by ideals (see for example [10]). In this subsection only, $R$ is a general commutative ring with the identity element 1 .

Let $M$ be an $R$-module and let $\left\{M_{n} \mid n=1,2, \ldots\right\}$ be a decreasing sequence of submodules of $M$. We have a topology on $M$ such that $\left\{x+M_{n} \mid n=1,2, \ldots\right\}$ is a fundamental system of neighborhoods of $x$ for any $x \in M$, and this topology is called the linear topology of $M$ defined by $\left\{M_{n} \mid n=1,2, \ldots\right\}$. For an ideal $I$ of $R$ the linear topology defined by $\left\{I^{n} M\right\}$ is called the $I$-adic topology. Especially, when $R=k[[\hbar]]$ and $I=(\hbar)$, the $I$-adic topology is called the $\hbar$-adic topology. Note that any homomorphism of $R$-modules is continuous with respect to the $I$-adic topologies.

An $R$-module $M$ with a linear topology is said to be separated if it is Hausdorff as a topological space, and is said to be complete if any Cauchy sequence has a limit in $M$. Here a sequence $\left\{x_{n}\right\}$ of elements of $M$ is called a Cauchy sequence if it satisfies the following condition:

(*) for any open submodule $L$ of $M$, there exists $n_{0}$ such that $x_{n 1}-x_{n 2} \in L$ for any $n_{1}, n_{2} \geqq n_{0}$.

Note that the limit of sequence in $M$ is not necessarily unique unless $M$ is separated.

Let $M$ be an $R$-module with a linear topology. An $R$-module $\hat{M}$ with a complete separated linear topology together with a continuous $R$-homomorphism $f: M \rightarrow \hat{M}$ is called the completion of $M$ if, for any $R$-module $L$ with a complete separated linear topology, and for any continuous $R$-homomorphism $f^{\prime}: M \rightarrow L$, there exists a unique continuous $R$-homomorphism $\varphi: \hat{M} \rightarrow L$ satisfying $\varphi \circ f=f^{\prime}$. It is known that the completion exists and is unique up to isomorphisms. When the linear topology of $M$ is defined by $\left\{M_{n}\right\}$, we have $\hat{M}=\lim M / M_{n}, \hat{M}_{n}=\operatorname{ker}\left(\hat{M} \rightarrow M / M_{n}\right)$, and $f: M \rightarrow \hat{M}$ is the natural homomorphism. It is easily seen that $f$ is injective (respectively surjective) if and only if $M$ is separated (respectively complete). The following is clear by definition:

Lemma 2.1.1. Let $M$ be an $R$-module with a linear topology and let $L$ be its submodule. If $f: M \rightarrow \hat{M}$ is the completion of $M$, then the natural homomorphism $M / L \rightarrow \hat{M} / \overline{f(L)}$ 
is the completion of $M / L$ with respect to the quotient topology of $M / L$. Here barring denotes the closure.

It is obvious that the quotient topology of $M / L$ with respect to the $I$-adic topology of $M$ coincides with the $I$-adic topology.

We will use the following lemma in Sect. 2.2. It is an easy consequence of the well-known fact that if $I$ is finitely generated, the topology of the $I$-adic completion $\hat{M}$ of an $R$-module $M$ is the $I$-adic topology.

Lemma. 2.1.2. Let $M$ be a separated $R$-module and let $M \hookrightarrow \hat{M}$ be the I-adic completion. If I is finitely generated, then the topology of $M$ induced from the I-adic topology of $\hat{M}$ coincides with the I-adic topology.

Example. Let $R=k[[\hbar]]$ be the formal power series ring over a field $k$. For a $k$-module $M_{0}$ set $M=R \otimes_{k} M_{0}$. Let $\hat{M}$ be the $\hbar$-adic completion of $M$. Then we have the following natural identifications:

$$
\begin{aligned}
& \hat{M}=\left\{\sum_{i=0}^{\infty} \hbar^{i} m_{i} \mid m_{i} \in M_{0}\right\}, \\
& M=\left\{\sum_{i=0}^{\infty} \hbar^{i} m_{i} \mid m_{i} \in M_{0}, \operatorname{dim}_{k}\left(\sum_{i=0}^{\infty} k m_{i}\right)<\infty\right\},
\end{aligned}
$$

where $\sum_{i=0}^{\infty} \hbar^{i} m_{i}$ is a formal infinite sum.

2.2. The Triangular Decomposition. In the rest of this paper $R$ denotes the formal power series ring $k[[\hbar]]$, where $k$ is a field of characteristic zero, and $R$-modules are endowed with the $\hbar$-adic topologies. We will use the notations in Sect. 1 . From now on until the end of Sect. 3 we assume that $A$ is a symmetrizable generalized Cartan matrix, which is not necessarily of finite type.

Let $I_{1}$ be the two-sided ideal of $\hat{U}$ generated by:

$$
\begin{gathered}
t t^{\prime}-t^{\prime} t \quad\left(t, t^{\prime} \in \mathrm{t}\right), \\
t e_{i}-e_{i} t-\alpha_{i}(t) e_{i} \quad(t \in \mathrm{t}, i=1, \ldots l), \\
t f_{i}-f_{i} t+\alpha_{i}(t) f_{i} \quad(t \in \mathrm{t}, i=1, \ldots, l), \\
e_{i} f_{j}-f_{j} e_{i}-\delta_{i, j} \frac{\sinh \left(\hbar t_{i} / 2\right)}{\sinh \left(\hbar d_{i} / 2\right)} \quad(i, j=1, \ldots l),
\end{gathered}
$$

and set $U_{1}=\hat{U} / \bar{I}_{1}$. Let $I_{2}$ be the two-sided ideal of $U_{1}$ generated by:

$$
\begin{aligned}
& \sum_{m=0}^{1-a_{1} j}(-1)^{m}\left[\begin{array}{c}
1-a_{i j} \\
m
\end{array}\right]_{q_{i}} e_{i}^{1-a_{i j}-m} e_{j} e_{i}^{m} \quad(i \neq j), \\
& \sum_{m=0}^{1-a_{i j}}(-1)^{m}\left[\begin{array}{c}
1-a_{i j} \\
m
\end{array}\right] f_{i}^{1-a_{i j}-m} f_{j} f_{i}^{m} \quad(i \neq j) .
\end{aligned}
$$

Lemma 2.2.1. The R-algebra $U_{h}(\mathfrak{g})(=\hat{U} / \bar{I})$ is naturally isomorphic to $U_{1} / \bar{I}_{2}$.

Proof. Let $f: \hat{U} \rightarrow U_{1}=\hat{U} / \bar{I}_{1}$ be the natural homomorphism. We have $f^{-1}(f(\bar{I}))=$ $\bar{I}$ by $\bar{I}_{1} \subset \bar{I}$. Since $f$ is a surjective open map and $f^{-1}(f(\bar{I}))$ is closed, we see that 
$f(\bar{I})$ is a closed subset of $U_{1}$, and hence we have $f(\bar{I})=\overline{f(I)}$. (Note that a surjective homomorphism of $R$-modules is automatically an open map with respect to the $\hbar$-adic topology.) Since $f(I)=I_{2}$, we have

$$
f^{-1}\left(\bar{I}_{2}\right)=f^{-1}\left(\overline{f(I))}=f^{-1}(f(\bar{I}))=\bar{I},\right.
$$

and the assertion is proved.

Let $R\left\langle x_{1}, \ldots, x_{l}\right\rangle$ (respectively $R\left\langle y_{1}, \ldots, y_{l}\right\rangle$ ) be the tensor algebra of the $R$-module with free basis $\left\{x_{1}, \ldots, x_{l}\right\}$ (respectively $\left\{y_{1}, \ldots, y_{l}\right\}$ ), and set

$$
V=R\left\langle y_{1}, \ldots, y_{l}\right\rangle \otimes_{R} S(\mathrm{t}) \otimes_{R} R\left\langle x_{1}, \ldots, x_{l}\right\rangle .
$$

Choose a basis $\left\{z_{1}, \ldots, z_{r}\right\}$ of the $k$-vector space $\mathrm{t}_{0}$. Then $V$ is a free $R$-module with basis:

$$
\left\{y_{i_{1}} \cdots y_{i_{p}} z_{1}^{a_{1}} \cdots z_{r}^{a_{r}} x_{j_{1}} \cdots x_{j_{q}} \mid p, q, a_{1}, \ldots, a_{r} \geqq 0, i_{1}, \ldots, i_{p}, j_{1}, \ldots, j_{q}=1, \ldots, l\right\} .
$$

We define a $\hat{U}$-module structure on the completion $\hat{V}$ by:

$$
\begin{aligned}
& e_{i} \cdot y_{i_{1}} \cdots y_{i_{p}} z_{1}^{a_{1}} \cdots z_{r}^{a_{r}} x_{j_{1}} \cdots x_{j_{q}} \\
& =\sum_{\substack{1 \leqq s \leqq p \\
i_{s}=i}} y_{i_{1}} \cdots \hat{y}_{i_{s}} \cdots y_{i_{p}} \frac{\sinh \left(\hbar\left(t_{i}-\left(\alpha_{i_{s}+1}+\cdots \alpha_{i_{p}}\right)\left(t_{i}\right)\right) / 2\right)}{\sinh \left(\hbar d_{i} / 2\right)} z_{1}^{a_{1}} \cdots z_{r}^{a_{r}} x_{j_{1}} \cdots x_{j_{q}} \\
& +y_{i_{1}} \cdots y_{i_{p}}\left(z_{1}-\alpha_{i}\left(z_{1}\right)\right)^{a_{1}} \cdots\left(z_{r}-\alpha_{i}\left(z_{r}\right)\right)^{a_{r}} x_{i} x_{j_{1}} \cdots x_{j_{q}} \quad(i=1, \ldots, l), \\
& t \cdot y_{i_{1}} \cdots y_{i_{p}} z_{1}^{a_{1}} \cdots z_{r}^{a_{r}} x_{j_{1}} \cdots x_{j_{q}} \\
& =y_{i_{1}} \cdots y_{i_{p}}\left(t-\left(\alpha_{i_{1}}+\cdots+\alpha_{i_{p}}\right)(t)\right) z_{1}^{a_{1}} \cdots z_{r}^{a_{r}} x_{j_{1}} \cdots x_{j_{q}} \quad(t \in \mathfrak{t}), \\
& f_{i} \cdot y_{i_{1}} \cdots y_{i_{p}} z_{1}^{a_{1}} \cdots z_{r}^{a_{r}} x_{j_{1}} \cdots x_{j_{q}} \\
& =y_{i} y_{i_{1}} \cdots y_{i_{p}} z_{1}^{a_{1}} \cdots z_{r}^{a_{r}} x_{j_{1}} \cdots x_{j_{q}} \quad(i=1, \ldots, l) .
\end{aligned}
$$

More precisely, we first define $R$-homomorphisms $V \rightarrow \hat{V}$ by the above formulas and extended them to the endomorphisms of the $R$-module $\hat{V}$. Since $U$ is a free $R$-algebra, we get an action of $U$ on $\hat{V}$, and it lifts uniquely to the action of $\hat{U}$ on $\hat{V}$.

Lemma 2.2.2. (i) The ideal $\bar{I}_{1}$ of $\hat{U}$ annihilates $\hat{V}$, and hence $\hat{V}$ is a $U_{1}$-module.

(ii) The R-homomorphism from $U_{1}$ to $\hat{V}$ given by $u \mapsto u \cdot 1$ is an isomorphism.

Proof. (i) Since $\hat{V}$ is separated, we have only to show $I_{1} V=0$. Details are omitted. (ii) Let $D$ be the $k$-subspace of $\hat{U}$ spanned by the elements

$$
f_{i_{1}} \cdots f_{i_{p}} z_{1}^{a} \cdots z_{r}^{a_{r}} e_{j_{1}} \cdots e_{j_{q}} \quad\left(p, q, a_{1}, \ldots, a_{r} \geqq 0, i_{1}, \ldots, i_{p}, j_{1}, \ldots, j_{q}=1, \ldots, l\right) .
$$

Then we have:

$$
\widehat{U}=I_{1}+D+\hbar \hat{U} \text {. }
$$

Indeed, this is easily proved using the fact that the $k$-algebra $\hat{U} /\left(I_{1}+\hbar \hat{U}\right)$ has a presentation with generators $\mathrm{t}_{0}, e_{1}, \ldots, e_{l}, f_{1}, \ldots, f_{l}$, and the fundamental relations:

$$
\begin{aligned}
t t^{\prime} & =t^{\prime} t \quad\left(t, t^{\prime} \in \mathrm{t}_{0}\right), \\
e_{i} t & =\left(t-\alpha_{i}(t)\right) e_{i} \quad\left(t \in \mathrm{t}_{0}, i=1, \ldots, l\right),
\end{aligned}
$$




$$
\begin{aligned}
t f_{i} & =f_{i}\left(t-\alpha_{i}(t)\right) \quad\left(t \in \mathrm{t}_{0}, i=1, \ldots, l\right), \\
e_{i} f_{j} & =f_{j} e_{i}+\delta_{i, j} t_{i} / d_{i} \quad(i, j=1, \ldots, l) .
\end{aligned}
$$

Let $u$ be an element of $\hat{U}$. By (2.2.11) we can take elements $b_{n} \in I_{1}, c_{n} \in D$, $e_{n} \in \hat{U}(n=0,1,2, \ldots)$ satisfying $u=b_{0}+c_{0}+\hbar e_{0}, e_{n}=b_{n+1}+c_{n+1}+\hbar e_{n+1}$. Then we have

$$
u=\sum_{i=0}^{n} \hbar^{i} b_{i}+\sum_{i=0}^{n} \hbar^{i} c_{i}+\hbar^{n+1} e_{n} \quad(n=0,1,2, \ldots) .
$$

Since $\hat{U}$ is complete and separated, any Cauchy sequence in $\hat{U}$ converges to a unique element, and hence we have:

$$
u=\sum_{i=0}^{\infty} \hbar^{i} b_{i}+\sum_{i=0}^{\infty} \hbar^{i} c_{i}
$$

Thus, setting $E=\left\{\sum_{i=0}^{\infty} \hbar^{i} c_{i} \mid c_{i} \in D\right\}$, we have;

$$
\widehat{U}=\bar{I}_{1}+E .
$$

Let $\varphi: \hat{U} \rightarrow \hat{V}$ be the $R$-homomorphism defined by $\varphi(u)=u \cdot 1$. By (i) we have $\varphi \mid \bar{I}_{1}=0$ and by definition we see that $\varphi \mid E$ is an isomorphism. Therefore the assertion follows from (2.2.12).

Let $N_{1}^{+}$(respectively $N_{1}^{-}$, respectively $T_{1}$ ) be the subalgebra of $U_{1}$ generated by $e_{1}, \ldots, e_{l}$ (respectively $f_{1}, \ldots, f_{l}$, respectively $\mathrm{t}$ ), and let $U_{1}^{f}$ be the subalgebra generated by $N_{1}^{+}, N_{1}^{-}, \bar{T}_{1}$.

Lemma 2.2.3. (i) We have isomorphisms of R-algebras:

$$
N_{1}^{+} \cong R\left\langle x_{1}, \ldots, x_{l}\right\rangle \cong N_{1}^{-} \quad\left(e_{i} \leftrightarrow x_{i} \leftrightarrow f_{i}\right) .
$$

(ii) $T_{1}$ is naturally isomorphic to the symmetric algebra $S(\mathrm{t})$ of the free $R$-module $\mathrm{t}$, and the inclusion $T_{1} \hookrightarrow \bar{T}_{1}$ is the $\hbar$-adic completion.

(iii) We have an isomorphism of $R$-modules:

$$
U_{1}^{f} \cong N_{1}^{+} \otimes \bar{T}_{1} \otimes N_{1}^{+} \quad(u v w \leftrightarrow u \otimes v \otimes w) .
$$

(iv) The inclusion $U_{1}^{f} \subseteq U_{1}$ is the $\hbar$-adic completion.

Proof. By Lemma 2.2.2 it is sufficient to show that $N_{1}^{-} \bar{T}_{1} N_{1}^{+}$is a subalgebra of $U_{1}$. This is easily checked by direct calculations.

Set

$$
\begin{aligned}
& u_{i j}^{+}=\sum_{m=0}^{1-a_{i j}}(-1)^{m}\left[\begin{array}{c}
1-a_{i j} \\
m
\end{array}\right]_{q_{i}} e_{i}^{1-a_{i j}-m} e_{j} e_{i}^{m} \in N_{1}^{+} \quad(i \neq j), \\
& u_{i j}^{-}=\sum_{m=0}^{1-a_{i j}}(-1)^{m}\left[\begin{array}{c}
1-a_{i j} \\
m
\end{array}\right]_{q_{i}} f_{i}^{1-a_{i j}-m} f_{j} f_{i}^{m} \in N_{1}^{-} \quad(i \neq j),
\end{aligned}
$$

and let $J^{+}$(respectively $J^{-}$, respectively $J$ ) be the two-sided ideal of $N_{1}^{+}$(respectively $N_{1}^{-}$, respectively $U_{1}^{f}$ ) generated by $\left\{u_{i j}^{+} \mid i \neq j\right\}$ (respectively $\left\{u_{i j}^{-} \mid i \neq j\right\}$, respectively $\left.\left\{u_{i j}^{+}, u_{i j}^{-} \mid i \neq j\right\}\right)$. 
Lemma 2.2.4. (i) $J=J^{-} \bar{T}_{1} N_{1}^{+}+N_{1}^{-} \bar{T}_{1} J^{+}$.

(ii) $\bar{J}=\bar{I}_{2}$.

(iii) $J=U_{1}^{f} \cap \bar{I}_{2}$.

(The bars denote the closures in $U_{1}$.)

Proof. (i) It is sufficient to show that $J^{-} \bar{T}_{1} N_{1}^{+}$and $N_{1}^{-} \bar{T}_{1} J^{+}$are two-sided ideals of $U_{1}^{f}$. Let us consider $J^{-} \bar{T}_{1} N_{1}^{+}$. Since it is apparently a right ideal, we have only to show that it is preserved under the left multiplications by $e_{i}, f_{i}(i=1, \ldots, l)$ and elements of $\bar{T}_{1}$. This can be shown by using the identity

$$
e_{n} u_{i j}^{-}=u_{i j}^{-} e_{n} \quad(i \neq j),
$$

which is proved by direct calculations. The proof for $N_{1}^{-} \bar{T}_{1} J^{+}$is the same.

(ii) Since $U_{1}^{f}$ is a dense subalgebra of $U_{1}, \bar{J}$ is a two-sided ideal of $U_{1}$, and hence we have $\bar{J} \supset \bar{I}_{2}$. Another inclusion $\bar{J} \subset \bar{I}_{2}$ is obvious.

(iii) By (ii) we have $U_{1}^{f} \cap \bar{I}_{2}=U_{1}^{f} \cap \bar{J}$. Since the topology of $U_{1}^{f}$ induced from the $I$-adic topology of $U_{1}$ coincides with the $I$-adic topology (Lemma 2.1.2), it is sufficient to show that $J$ is closed in $U_{1}^{f}$ with respect to the $I$-adic topology of $U_{1}^{f}$. Under the identification $U_{1}^{f} \cong N_{1}^{-} \otimes \bar{T}_{1} \otimes N_{1}^{+}$of Lemma 2.2.3 (iii), $J$ corresponds to $J^{-} \otimes \bar{T}_{1} \otimes N_{1}^{+}+N_{1}^{-} \otimes \bar{T}_{1} \otimes J^{+}$by (i). (Since $R$ is a principal ideal domain, an $R$-module is flat if and only if it is torsion free. Hence $J^{-} \otimes \bar{T}_{1} \otimes N_{1}^{+}$and $N_{1}^{-} \otimes \bar{T}_{1} \otimes J^{+}$are naturally identified with submodules of $N_{1}^{-} \otimes \bar{T}_{1} \otimes N_{1}^{+}$.)

For an $l$-tuple $H=\left(h_{1}, \ldots, h_{l}\right)$ of non-negative integers, let $N_{1, H}^{+}$(respectively $N_{1, H}^{-}$) be the $R$-submodule of $N_{1}^{+}$(respectively $N_{1}^{-}$) spanned by the elements $e_{i_{1}} \cdots e_{i_{p}}$ (respectively $\left.f_{i_{1}} \cdots f_{i_{p}}\right)$ such that $i$ appears $h_{i}$-times in the sequence $\left(i_{1}, \ldots, i_{p}\right)$ for $i=1, \ldots, l$. Setting $J_{H}^{ \pm}=J^{ \pm} \cap N_{1, H}^{ \pm}$, we have $N_{1}^{ \pm}=\oplus_{H} N_{1, H}^{ \pm}$and $J^{ \pm}=\oplus_{H} J_{H}^{ \pm}$by the definition of $J^{ \pm}$. Hence we have:

$$
\begin{aligned}
N_{1}^{-} \otimes \bar{T}_{1} \otimes N_{1}^{+} & =\oplus_{H, H^{\prime}}\left(N_{1, H}^{-} \otimes \bar{T}_{1} \otimes N_{1, H^{\prime}}^{+}\right), \\
J^{-} \otimes \bar{T}_{1} \otimes N_{1}^{+}+N_{1}^{-} \otimes \bar{T}_{1} \otimes J^{+} & =\oplus_{H, H^{\prime}}\left(J_{H}^{-} \otimes \bar{T}_{1} \otimes N_{1, H^{\prime}}^{+}+N_{1, H}^{-} \otimes \bar{T}_{1} \otimes J_{H^{\prime}}^{+}\right) .
\end{aligned}
$$

Therefore it is sufficient to show that $\left(J_{H}^{-} \otimes N_{1, H^{\prime}}^{+}+N_{1, H}^{-} \otimes J_{H^{\prime}}^{+}\right) \otimes \bar{T}_{1}$ is closed in $\left(N_{1, H}^{-} \otimes N_{1, H^{\prime}}^{+}\right) \otimes \bar{T}_{1}$ for each $H, H^{\prime}$. Since $N_{1, H}^{-} \otimes N_{1, H^{\prime}}^{+}$is a free $R$-module of finite rank, we have only to show that $(0)$ and $\hbar^{n} \bar{T}_{1}(n=0,1,2, \ldots)$ are closed in $\bar{T}_{1}$. This is obvious by definition.

Proof of Theoem 1 Except for the Freeness of $N$. By Lemma 2.2.1 and Lemma 2.2 .4 (ii) we have $U_{\hbar}^{f}(\mathfrak{g}) \cong U_{1}^{f} / J$. Hence the statements (i), (ii), (iii) except for the freeness of $N$ follow from Lemma 2.2.3 (i), (ii), (iii) and Lemma 2.2.4(i). The statement (iv) is a consequence of Lemma 2.2.3 (iv), Lemma 2.2.4 (ii), Lemma 2.2.1 and Lemma 2.1.1.

Set

$$
\begin{gathered}
Q=\left\{\sum_{i=1}^{l} m_{i} \alpha_{i} \mid m_{i} \in Z\right\} \subset \mathrm{t}_{0}^{*}, \\
Q^{+}=\left\{\sum_{i=1}^{l} m_{i} \alpha_{i} \in Q \mid m_{i} \geqq 0\right\} \subset \mathrm{t}_{0}^{*} .
\end{gathered}
$$


Note that $\left\{\alpha_{1}, \ldots, \alpha_{l}\right\}$ is linearly independent. For $\gamma=\sum_{i=1}^{l} m_{i} \alpha_{i} \in Q^{+}$, let $N_{\gamma}^{+}$ (respectively $N_{-\gamma}^{-}$) be the $R$-submodule of $N^{+}$(respectively $N^{-}$) generated by the elements $e_{i_{1}} \cdots e_{i_{p}}$ (respectively $f_{i_{1}} \cdots f_{i_{p}}$ ) such that $i$ appears $m_{i}$-times in the sequence $\left(i_{1}, \ldots, i_{p}\right)$. By definition we have the following:

$$
N^{ \pm}=\bigoplus_{\gamma \in Q^{+}} N_{ \pm \gamma}^{ \pm}
$$

\section{Highest Weight Modules}

3.1. Highest Weight Modules. Let $K=k((\hbar))$ denote the quotient field of $R=k[[\hbar]]$. Set

$$
\begin{aligned}
\tilde{U}_{\hbar}^{f}(\mathfrak{g}) & =K \otimes_{R} U_{\hbar}^{f}(\mathrm{~g}), \\
\tilde{N}^{ \pm} & =K \otimes_{R} N^{ \pm}, \quad \tilde{N}_{ \pm \gamma}^{ \pm}=K \otimes_{R} N_{ \pm \gamma}^{ \pm} \quad\left(\gamma \in Q^{+}\right) .
\end{aligned}
$$

By (2.2.18) we have:

$$
\tilde{N}^{ \pm}=\bigoplus_{\gamma \in Q^{+}} \tilde{N}_{ \pm \gamma}^{ \pm} .
$$

For $\lambda \in \operatorname{Hom}_{R}(\mathrm{t}, R)$ let $\xi_{\lambda}: \bar{T} \rightarrow R$ be the $R$-algebra homomorphism determined by $\xi_{\lambda}(t)=\lambda(t)(t \in \mathfrak{t})$. We denote by $\tilde{\xi}_{\lambda}: K \otimes_{R} \bar{T} \rightarrow K$ the scalar extension of $\xi_{\lambda}$.

Let $\lambda \in \operatorname{Hom}_{R}(\mathrm{t}, R) . A \tilde{U}_{h}^{f}(\mathfrak{g})$-module $M$ is called a highest weight module with highest weight $\lambda$ if $M$ is generated by a non-zero element $v \in M$ satisfying $e_{i} \cdot v=0$ $(i=1, \ldots, l), t \cdot v=\widetilde{\xi}_{\lambda}(t) v\left(t \in K \otimes_{R} \bar{T}\right)$. Such $v$ is called a highest weight vector.

For a $\tilde{U}_{h}^{f}(\mathrm{~g})$-module $M$ and $\mu \in \operatorname{Hom}_{R}(\mathrm{t}, R)$ set

$$
M_{\mu}=\left\{m \in M \mid t \cdot m=\tilde{\xi}_{\mu}(\mathrm{t}) m \text { for } t \in K \otimes_{R} \bar{T}\right\} .
$$

We define an ordering on $\operatorname{Hom}_{R}(\mathrm{t}, R)$ by

$$
\lambda \geqq \mu \quad \text { if and only if } \lambda-\mu \in Q^{+} .
$$

Lemma 3.1.1. Let $M$ be a highest weight module of $\tilde{U}_{\hbar}^{f}(\mathfrak{g})$ with highest weight $\lambda \in \operatorname{Hom}_{R}(\mathrm{t}, R)$ and let $v$ be a highest weight vector.

(i) $M=\bigoplus_{\mu \leq \lambda} M_{\mu}$ and $M_{\lambda-\gamma}=\tilde{N}_{-\gamma}^{-} v\left(\gamma \in Q^{+}\right)$.

(ii) $M$ has a unique irreducible quotient, which is also a highest weight module with highest weight $\lambda$.

(iii) Let $M^{\prime}$ be the $U_{h}^{f}(\mathrm{~g})$-submodule of $M$ generated by $v$. Then we have

$$
M^{\prime}=\bigoplus_{\mu \leqq \lambda}\left(M^{\prime} \cap M_{\mu}\right), \quad M^{\prime} \cap M_{\lambda-\gamma}=N_{-\gamma}^{-} v \quad\left(\gamma \in Q^{+}\right) .
$$

Furthermore, $M^{\prime} \cap M_{\mu}$ is a free $R$-module of finite rank and the natural map $K \otimes_{R}\left(M^{\prime} \cap M_{\mu}\right) \rightarrow M_{\mu}$ is an isomorphism. Here $M^{\prime}$ is a free $R$-module and we have $K \otimes_{R} M^{\prime} \cong M$.

Proof. (i) This follows from the following obvious facts:

$$
M=\tilde{N}^{-} v=\sum_{\gamma \in Q+} \tilde{N}_{-\gamma}^{-} v, \quad \tilde{N}_{-\gamma}^{-} v \subset M_{\lambda-\gamma}
$$


(ii) It follows from (i) that any submodule of $M$ is a direct sum of certain subspaces of $M_{\lambda-\gamma}=\tilde{N}_{-\gamma}^{-} v\left(\gamma \in Q^{+}\right)$. Hence $M$ has a unique maximal submodule contained in $\bigoplus_{\mu<\lambda} M_{\mu}$.

(iii) The first half is clear. Let $f: K \otimes\left(M^{\prime} \cap M_{\mu}\right) \rightarrow M_{\mu}$ be the natural map. Since $M^{\prime} \cap M_{\mu}$ is a finitely generated $R$-submodule of the $K$-vector space $M_{\mu}$, and since $f$ is surjective, we see that $f$ is an isomorphism.

For $\lambda \in \operatorname{Hom}_{R}(\mathrm{t}, R)$ we set

$$
\tilde{M}^{f}(\lambda)=\tilde{U}_{h}^{f}(\mathfrak{g}) /\left(\sum_{i=1}^{l} \tilde{U}_{\hbar}^{f}(\mathfrak{g}) e_{i}+\tilde{U}_{\hbar}^{f}(\mathfrak{g}) \operatorname{ker} \xi_{\lambda}\right) .
$$

This is obviously a highest weight module with highest weight $\lambda$. Since any highest weight module with highest weight $\lambda$ is a quotient of $\tilde{M}^{f}(\lambda)$, there exists a unique irreducible highest weight module $\tilde{L}^{f}(\lambda)$ with highest weight $\lambda$. We denote by $M^{f}(\lambda)$ (respectively $L^{f}(\lambda)$ ) the $U_{h}^{f}(\mathfrak{g})$-submodule of $\tilde{M}^{f}(\lambda)$ (respectively $\tilde{L}^{f}(\lambda)$ ) generated by a highest weight vector. We call $\tilde{M}^{f}(\lambda)$ and $M^{f}(\lambda)$ the Verma modules.

A $\tilde{U}_{h}^{f}(\mathfrak{g})$-module $M$ is said to be integrable if the elements $e_{i}, f_{i}(i=1, \ldots, l)$ act on $M$ locally nilpotently. The following is proved in exactly the same manner as in [9].

Lemma 3.1.2. Let $\lambda$ be an element of $\operatorname{Hom}_{R}(\mathrm{t}, R)$. Then $\tilde{L}^{f}(\lambda)$ is integrable if and only if $\lambda\left(2 t_{i} / \alpha_{i}\left(t_{i}\right)\right)$ is a non-negative integer for each $i=1, \ldots, l$.

For $\lambda \in \operatorname{Hom}_{R}(\mathrm{t}, R)$ we define $\lambda^{0} \in \mathrm{t}_{0}^{*}$ by:

$$
\lambda^{0}(t)=\left.\lambda(t)\right|_{h=0} \quad\left(t \in \mathfrak{t}_{0}\right) .
$$

Let $\lambda$ be an element of $\operatorname{Hom}_{R}(\mathrm{t}, R)$ such that $\lambda\left(2 t_{i} / \alpha_{i}\left(t_{i}\right)\right)$ is a non-negative integer for $i=1, \ldots, l$. Then as in [9] we can determine the dimensions of the spaces $\tilde{L}^{f}(\lambda)_{\mu}$ for $\mu \leqq \lambda$ in the following manner. Set $L^{0}(\lambda)=k \otimes_{R} L^{f}(\lambda)$, where the ring homomorphism $R \rightarrow k$ is given by $\hbar \rightarrow 0$. Since $k \otimes_{R} U_{\hbar}^{f}(\mathfrak{g})\left(=U_{\hbar}^{f}(\mathfrak{g}) / \hbar U_{h}^{f}(\mathfrak{g})\right)$ is naturally isomorphic to the universal enveloping algebra $U(\mathrm{~g})$ of the Kac-Moody Lie algebra $g$, we have a $U(\mathfrak{g})$-module structure on $L^{0}(\lambda)$. Furthermore it is easily seen that $L^{0}(\lambda)$ is the integrable highest weight module with highest weight $\lambda^{0}$ in the sense of [8]. Identify $t_{0}$ with the abelian subalgebra of $g$ and set

$$
L^{0}(\lambda)_{v}=\left\{m \in L^{0}(\lambda) \mid t \cdot m=v(t) m \quad\left(t \in \mathrm{t}_{0}\right)\right\} \quad\left(v \in \mathfrak{t}_{0}^{*}\right) .
$$

Then by Lemma 3.1 .1 (iii) we see that, for $\gamma \in Q^{+}, \operatorname{dim}_{K} \tilde{L}^{f}(\lambda)_{\lambda-\gamma}$ coincides with $\operatorname{dim}_{k} L^{0}(\lambda)_{\bar{\lambda}-\gamma}$, and this is given by the Weyl-Kac formula ([8]).

In order to write down the formula explicitly we need some notations concerning the Kac-Moody Lie algebra $\mathfrak{g}$.

Set

$$
\begin{aligned}
\mathfrak{g}_{\alpha} & =\left\{x \in \mathfrak{g} \mid(\operatorname{ad}(t))(x)=\alpha(t) x\left(t \in \mathrm{t}_{0}\right)\right\} \quad(\alpha \in Q), \\
\Delta & =\left\{\alpha \in Q-(0) \mid \mathfrak{g}_{\alpha} \neq(0)\right\}, \quad \Delta^{+}=\Delta \cap Q^{+}, \\
W & \left.=\left\langle s_{i} \mid i=1, \ldots, l\right\rangle \subset G L\left(\mathrm{t}_{0}\right) \quad \text { with } \quad s_{i}(t)=t-\left(2 \alpha_{i}(t)\right) / \alpha_{i}\left(t_{i}\right)\right) t_{i} .
\end{aligned}
$$

Then it is known that we have

$$
\Delta=\Delta^{+} \cup\left(-\Delta^{+}\right), \quad \operatorname{dim} \mathfrak{g}_{\alpha}=\operatorname{dim} \mathfrak{g}_{-\alpha}<\infty,
$$


and that $\Delta$ is preserved under the contragredient action of $W$. Let $\rho$ be an element of $t_{0}^{*}$ satisfying $\rho\left(2 t_{i} / \alpha_{i}\left(t_{i}\right)\right)=1$ for $i=1, \ldots, l$. Set

$$
l(w)=\min \left\{p \mid w=s_{i_{1}} \cdots s_{i_{p}} \text { for some } i_{1}, \ldots, i_{p} \in[1, l]\right\}
$$

for $w \in W$. Then the preceding arguments imply the following:

Lemma 3.1.3 ([9]). Let $\lambda$ be an element of $\operatorname{Hom}_{R}(\mathrm{t}, R)$ such that $\lambda\left(2 t_{i} / \alpha_{i}\left(t_{i}\right)\right)$ is a non-negative integer for each $i=1, \ldots, l$. Then we have:

$$
\sum_{\mu \leqq \lambda} \operatorname{dim} \tilde{L}^{f}(\lambda)_{\mu} e^{\mu}=\frac{\sum_{w \in W}(-1)^{l(w)} e^{w(\lambda+\rho)-\rho}}{\prod_{\alpha \in \Delta^{+}}\left(1-e^{-\alpha}\right)^{\operatorname{dim} g_{\alpha}}} .
$$

3.2. Freeness of $N$. Let us show that $N$ is a free $R$-module. By (1.15) and (2.2.18) it is sufficient to show that $N_{-\gamma}^{-}$is a free $R$-module for each $\gamma \in Q^{+}$. Since $N_{-\gamma}^{-}$is a finitely generated $R$-module, we have:

$$
N_{-\gamma}^{-} \cong R^{n} \oplus\left(\bigoplus_{j=1}^{m} R /\left(\hbar^{p_{J}}\right)\right)
$$

for some $n, m \geqq 0, p_{1}, \ldots, p_{m} \geqq 1$. Then we have $\tilde{N}_{-\gamma}^{-}=K \otimes_{R} N_{-\gamma}^{-} \cong K^{n}$ and $k \otimes_{R} N_{-\gamma}^{-} \cong k^{n+m}$. Hence we have only to show

$$
\operatorname{dim}_{K} \tilde{N}_{-\gamma}^{-} \geqq \operatorname{dim}_{k}\left(k \otimes_{R} N_{-\gamma}^{-}\right) .
$$

Let $M$ be a highest weight module of $\tilde{U}_{h}^{f}(\mathfrak{g})$ with highest weight $\lambda \in \operatorname{Hom}_{R}(\mathrm{t}, R)$. By Lemma 3.1.1 (i) we have:

$$
\operatorname{dim}_{K} \tilde{N}_{-\gamma}^{-} \geqq \operatorname{dim}_{K} M_{\lambda-\gamma} .
$$

On the other hand, since $k \otimes_{R} N^{-}$is naturally isomorphic to the enveloping algebra of the Lie subalgebra $\bigoplus_{\alpha \in \Delta^{+}} \mathfrak{g}_{-\alpha}$ of $\mathfrak{g}$, we see by the Poincaré-Birkhoff-Witt theorem that

$$
\operatorname{dim}_{k}\left(k \otimes_{R} N_{-\gamma}^{-}\right)=\text {the coefficient of } e^{-\gamma} \text { in } \frac{1}{\prod_{\alpha \in \Delta^{+}}\left(1-e^{-\alpha}\right)^{\operatorname{dim} g_{\alpha}}} .
$$

Hence it suffices to show that there exist some $\lambda \in \operatorname{Hom}_{R}(\mathrm{t}, R)$ and a highest weight module $M$ of $\tilde{U}_{h}^{f}(\mathfrak{g})$ with highest weight $\lambda$ such that

$$
\operatorname{dim}_{K} M_{\lambda-\gamma}=\text { the coefficient of } e^{-\gamma} \text { in } \frac{1}{\prod_{\alpha \in \Delta^{+}}\left(1-e^{-\alpha}\right)^{\operatorname{dim} g_{\alpha}}} .
$$

Let $\gamma=\sum_{i=1}^{l} m_{i} \alpha_{i} \in Q^{+}$. Let $\lambda$ be an element of $\operatorname{Hom}_{R}(\mathrm{t}, R)$ satisfying $\lambda\left(2 t_{i} / \alpha_{i}\left(t_{i}\right)\right)=$ $m_{i}(i=1, \ldots, l)$, and set $M=\tilde{L}^{f}(\lambda)$. By Proposition 3.1.3 we have

$$
\operatorname{dim}_{K} M_{\lambda-\gamma}=\text { the coefficient of } e^{\lambda-\gamma} \text { in } \frac{\sum_{w \in W}(-1)^{l(w)} e^{w(\lambda+\rho)-\rho}}{\prod_{\alpha \in \Delta^{+}}\left(1-e^{-\alpha}\right)^{\operatorname{dim} g_{\alpha}}}
$$




$$
\begin{aligned}
= & \text { the coefficient of } e^{-\gamma} \text { in } \frac{1}{\prod_{\alpha \in \Delta^{+}}\left(1-e^{-\alpha}\right)^{\mathrm{dimg}_{\alpha}}}+\sum_{\substack{w \in W \\
w \neq 1}}(-1)^{l(w)} \\
& \cdot\left(\text { the coefficient of } e^{-(\gamma+w(\lambda+\rho)-(\lambda+\rho))} \frac{1}{\prod_{\alpha \in \Delta^{+}}\left(1-e^{-\alpha}\right)^{\mathrm{dimg}_{\alpha}}}\right),
\end{aligned}
$$

and hence it is sufficient to show

$$
\gamma+w(\lambda+\rho)-(\lambda+\rho) \notin Q^{+} \quad(w \in W, w \neq 1) .
$$

Let us prove (3.2.5) by induction on $l(w)$. If $l(w)=1$, we have $w=s_{j}$ for some $j$, and hence

$$
\gamma+w(\lambda+\rho)-(\lambda+\rho)=\gamma-\left(m_{j}+1\right) \alpha_{j} \notin Q^{+} .
$$

Assume that $l(w) \geqq 2$. There exist some $j$ and $y \in W$ such that $w=s_{j} y$ with $l(y)=l(w)-1$, and then it is known that $y^{-1}\left(\alpha_{j}\right) \in \Delta^{+}$(see [8]). We have

$$
\gamma+w(\lambda+\rho)-(\lambda+\rho)=(\gamma+y(\lambda+\rho)-(\lambda+\rho))-(\lambda+\rho)\left(y^{-1}\left(2 t_{j} / \alpha_{j}\left(t_{j}\right)\right)\right) \alpha_{j},
$$

and $y^{-1}\left(2 t_{j} / \alpha_{j}\left(t_{j}\right)\right)$ is a linear combination of $2 t_{i} / \alpha_{i}\left(t_{i}\right)(i=1, \ldots, l)$ with non-negative integral coefficients. Hence we have (3.2.5), and the freeness of $N$ is verified.

The proof of Theorem 1 is completed.

Corollary 3.2.1 ([3]). (i) $U_{h}(\mathrm{~g})$ is topologically free; i.e., it is the completion of a free $R$-module.

(ii) $U_{h}(\mathrm{~g})$ is an integral domain; i.e., if $x, y$ are elements of $U_{h}(\mathrm{~g})$ such that $x y=0$, we have $x=0$ or $y=0$.

Proof. (i) By Theorem $1 U_{h}(\mathrm{~g})$ is the completion of the free $R$-module $N^{-} \otimes T \otimes N^{+}$. (ii) This follows from (i) and the fact that $U_{h}(\mathfrak{g}) / \hbar U_{h}(\mathfrak{g})(\cong U(\mathfrak{g}))$ is an integral domain.

3.3. Verma Modules. For $\lambda \in \operatorname{Hom}_{R}(\mathrm{t}, R)$ we define a $U_{\hbar}(\mathrm{g})$-module $M(\lambda)$ to be the $\hbar$-adic completion of the $U_{\hbar}^{f}(\mathrm{~g})$-module $M^{f}(\lambda)$.

Lemma 3.3.1. Let $\lambda \in \operatorname{Hom}_{R}(\mathrm{t}, R)$ and let $v$ be a highest weight vector of $M^{f}(\lambda)$.

(i) The R-homomorphism $N^{-} \rightarrow M^{f}(\lambda)(u \mapsto u \cdot v)$ is an isomorphism.

(i)' The R-homomorphism $\overline{N^{-}} \rightarrow M(\lambda)(u \mapsto u \cdot v)$ is an isomorphism.

(ii) $M^{f}(\lambda) \cong U_{\hbar}^{f}(\mathrm{~g}) /\left(\sum_{i=1}^{l} U_{\hbar}^{f}(\mathrm{~g}) e_{i}+U_{\hbar}^{f}(\mathrm{~g}) \operatorname{ker} \xi_{\lambda}\right)$.

(ii) $^{\prime} M(\lambda) \cong U_{\hbar}(\mathfrak{g}) \sqrt{\left(\sum_{i=1}^{l} U_{\hbar}(\mathfrak{g}) e_{i}+U_{\hbar}(\mathfrak{g}) \operatorname{ker} \tilde{\xi}_{\lambda}\right)}$.

Proof. By Theorem 1 we have

$$
\begin{aligned}
& U_{h}^{f}(\mathfrak{g})=N^{-} \oplus\left(\sum_{i=1}^{l} U_{\hbar}^{f}(\mathfrak{g}) e_{i}+U_{\hbar}^{f}(\mathfrak{g}) \operatorname{ker} \xi_{\lambda}\right), \\
& \tilde{U}_{\hbar}^{f}(\mathfrak{g})=\tilde{N}^{-} \oplus\left(\sum_{i=1}^{l} \tilde{U}_{\hbar}^{f}(\mathfrak{g}) e_{i}+\tilde{U}_{\hbar}^{f}(\mathfrak{g}) \operatorname{ker} \tilde{\xi}_{\lambda}\right) .
\end{aligned}
$$


Since $N^{-}$is a free $R$-module, the natural map $N^{-} \rightarrow \tilde{N}^{-}=K \otimes_{R} N^{-}$is injective, and hence we have (i), (ii). The statements (i)', (ii)' follow from Theorem 1 and Lemma 2.1.1

Lemma 3.3.2. Let $\lambda \in \operatorname{Hom}_{R}(\mathrm{t}, R)$ and $i \in[1, l]$. Set $m=\lambda\left(2 t_{i} / \alpha_{i}\left(t_{i}\right)\right)+1$. If $m$ is $a$ non-negative integer, then $M\left(\lambda-m \alpha_{i}\right)$ is isomorphic to a $U_{\hbar}(\mathrm{g})$-submodule of $M(\lambda)$.

Proof. Let $v$ be a highest weight vector of $M^{f}(\lambda)$ and set $v_{1}=f_{i}^{m} \cdot v$. By a direct calculation it is seen that $e_{j} \cdot v_{1}=0(j=1, \ldots, l)$ and $t \cdot v_{1}=\xi_{\lambda-m \alpha_{i}}(t) v_{1}(t \in \bar{T})$. Hence we have a $U_{h}^{f}(\mathfrak{g})$-homomorphism $M^{f}\left(\lambda-m \alpha_{i}\right) \rightarrow M^{f}(\lambda)$, sending a highest weight vector to $v_{1}$. Taking the completions we get a $U_{h}(\mathrm{~g})$-homomorphism $M\left(\lambda-m \alpha_{i}\right) \rightarrow$ $M(\lambda)$. The injectivity of this homomorphism follows from Lemma 3.3.1 (i)' and Corollary 3.2.1 (ii).

\section{Center}

In this section $A$ is a Cartan matrix of finite type and we take $\mathrm{t}_{0}$ to be the linear hull of $t_{1}, \ldots, t_{l}$.

4.1. Harish-Chandra Homomorphism. The purpose of this subsection is to prove the following.

Proposition 4.1.1. The R-homomorphism $\delta: \jmath\left(U_{\hbar}(\mathfrak{g})\right) \rightarrow \bar{T}$ defined in Sect. 1 is an algebra homomorphism and its image is contained in $\bar{T}^{W}$.

The following arguments are parallel to those for $U(\mathfrak{g})$ (see [2]).

By Theorem 1 we have

$$
U_{h}^{f}(\mathfrak{g})=\bar{T} \oplus\left(\sum_{i=1}^{l} U_{h}^{f}(\mathfrak{g}) e_{i}+\sum_{i=1}^{l} f_{i} U_{h}^{f}(\mathfrak{g})\right),
$$

and hence

$$
U_{h}(\mathfrak{g})=\bar{T} \oplus \overline{\left.\sum_{i=1}^{l} U_{h}(\mathfrak{g}) e_{i}+\sum_{i=1}^{l} f_{i} U_{h}(\mathfrak{g})\right)} .
$$

Let $p: U_{h}(\mathrm{~g}) \rightarrow \bar{T}$ be the projection with respect to (4.1.2). Let $\beta: \bar{T} \rightarrow \bar{T}$ and $\hat{\delta}: U_{h}(\mathfrak{g}) \rightarrow \bar{T}$ be as in Sect. 1. Then we have $\hat{\delta}=\beta \circ p$ by definition.

Lemma 4.1.2. For $z \in_{3}\left(U_{h}(\mathfrak{g})\right)$ we have

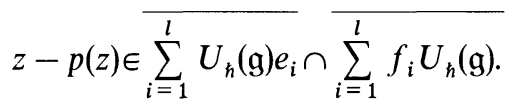

Proof. For $\gamma \in Q$ set

$$
U_{h}^{f}(\mathfrak{g})_{\gamma}=\bigoplus_{\substack{\gamma_{1}, \gamma_{2} \in Q^{+} \\ \gamma_{2}-\gamma_{1}=\gamma}}\left(N_{-\gamma_{1}}^{-} \otimes \bar{T} \otimes N_{\gamma_{2}}^{+}\right) \subset U_{\hbar}^{f}(\mathfrak{g}) .
$$

Then we have

$$
\begin{gathered}
U_{\hbar}^{f}(\mathfrak{g})=\bigoplus_{\gamma \in \mathcal{Q}} U_{h}^{f}(\mathfrak{g})_{\gamma}, \\
t x-x t=\gamma(t) x \quad\left(t \in \mathfrak{t}, x \in U_{h}^{f}(\mathfrak{g})_{\gamma}\right) .
\end{gathered}
$$


Set $z_{n}=\left(z \bmod \hbar^{n}\right) \in U_{h}(\mathfrak{g}) / \hbar^{n} U_{\hbar}(\mathfrak{g})=U_{\hbar}^{f}(\mathfrak{g}) / \hbar^{n} U_{\hbar^{\prime}}^{f}(\mathfrak{g})$. Since $z_{n}$ commutes with any element of $\mathrm{t} / \hbar^{n} \mathrm{t}$, we have $z_{n} \in U_{\hbar}^{f}(\mathrm{~g})_{0} / \hbar^{n} U_{\hbar}^{f}(\mathrm{~g})_{0}$ by (4.1.3) and (4.1.4). Thus we have $z \in \overline{U_{h}^{f}(\mathfrak{g})_{0}}$. By the definition of $U_{h}^{f}(\mathfrak{g})$ we have

$$
y-p(y) \in \sum_{i=1}^{l} U_{h}^{f}(\mathrm{~g}) e_{i} \cap \sum_{i=1}^{l} f_{i} U_{\hbar}^{f}(\mathfrak{g}) \quad\left(y \in U_{\hbar}^{f}(\mathfrak{g})_{0}\right),
$$

and hence

$$
y-p(y) \in \overline{\sum_{i=1}^{l} U_{h}(\mathfrak{g}) e_{i}} \cap \overline{\sum_{i=1}^{l} f_{i} U_{h}(\mathfrak{g})} \quad\left(y \in \overline{U_{h}^{f}(\mathfrak{g})_{0}}\right) .
$$

We are done.

Lemma 4.1.3. Let $\lambda \in \operatorname{Hom}_{R}(\mathrm{t}, R)$. For $z \in \mathfrak{\jmath}\left(U_{h}(\mathfrak{g})\right)$ and $m \in M(\lambda-\rho)$ we have $z \cdot m=$ $\xi_{\lambda}(\delta(z)) m$.

Proof. Let $v$ be a highest weight vector of $M(\lambda-\rho)$. Since $e_{i} \cdot v=0(i=1, \ldots, l)$, we have $(z-p(z)) \cdot v=0$ by Lemma 4.1.2, and hence we have

$$
z \cdot v=p(z) v=\xi_{\lambda-\rho}(p(z)) v=\xi_{\lambda}(\delta(z)) v .
$$

Since any element $m \in M(\lambda-\rho)$ is of the form $m=u \cdot v\left(u \in U_{h}(\mathfrak{g})\right)$, we have

$$
z \cdot m=(z u) \cdot v=(u z) \cdot v=u \cdot(z \cdot v)=\xi_{\lambda}(\delta(z)) u v=\xi_{\lambda}(\delta(z)) m .
$$

Let $P$ be the set of elements $\lambda \in \mathrm{t}_{0}^{*}$ such that $\lambda\left(2 t_{i} / \alpha_{i}\left(t_{i}\right)\right)$ is an integer for each $i=1, \ldots, l$. This is a lattice in $\mathrm{t}_{0}^{*}$ preserved under $W$.

Lemma 4.1.4. If $\lambda, \mu \in P$ are in the same $W$-orbit, then we have

$$
\xi_{\lambda}(\delta(z))=\xi_{\mu}(\delta(z)) \quad\left(z \in \mathfrak{z}\left(U_{h}(\mathfrak{g})\right)\right) .
$$

Proof. We may assume that $\lambda=s_{i} \mu$. Since $\lambda\left(2 t_{i} / \alpha_{i}\left(t_{i}\right)\right)=-\mu\left(2 t_{i} / \alpha_{i}\left(t_{i}\right)\right)$, we may also assume that $\lambda\left(2 t_{i} / \alpha_{i}\left(t_{i}\right)\right)$ is a non-negative integer. Then by Lemma 3.3.2 we see that $M(\mu-\rho)$ is isomorphic to a submodule of $M(\lambda-\rho)$. Since $M(\lambda-\rho)$ is a torsion free $R$-module by Theorem 1 and Lemma 3.3.1, the assertion follows from Lemma 4.1.3.

Proof of Proposition 4.1.1. Let $z_{1}, z_{2} \in \mathfrak{z}\left(U_{h}(\mathfrak{g})\right)$. Then by Lemma 4.1.2 we have

$$
z_{1}=p\left(z_{1}\right)+y_{1}, \quad z_{2}=p\left(z_{2}\right)+y_{2} \quad\left(y_{1}, y_{2} \in \overline{\sum_{i=1}^{l} U_{h}(\mathfrak{g}) e_{i}} \cap \overline{\sum_{i=1}^{l} f_{i} U_{h}(\mathfrak{g})}\right) .
$$

Since $y_{1} p\left(z_{2}\right)+p\left(z_{1}\right) y_{2}+y_{1} y_{2}$ is an element of $\overline{\sum_{i=1}^{l} U_{h}(\mathfrak{g}) e_{i}}+\overline{\sum_{i=1}^{l} f_{i} U_{h}(\mathfrak{g})}$, we have $p\left(z_{1} z_{2}\right)=p\left(z_{1}\right) p\left(z_{2}\right)$, and hence $p: z\left(U_{h}(\mathfrak{g})\right) \rightarrow \bar{T}$ is an algebra homomorphism. Since $\delta(z)=\beta(p(z))$ for $z \in \mathfrak{z}\left(U_{h}(\mathfrak{g})\right)$, we conclude that $\delta$ is an algebra homomorphism. Let us show that $\delta(z)=w \cdot \delta(z)$ for $z \in \mathfrak{z}\left(U_{h}(\mathrm{~g})\right)$ and $w \in W$. For any $\lambda \in P$, we have

$$
\xi_{\lambda}(\delta(z)-w \cdot \delta(z))=\xi_{\lambda}(\delta(z))-\xi_{w^{-1}(\lambda)}(\delta(z))=0,
$$

by Lemma 4.1.4. Set $\delta(z)-w \cdot \delta(z)=\sum_{i=0}^{\infty} \hbar^{i} y_{i}\left(y_{i} \in S\left(\mathrm{t}_{0}\right)\right)$. Then we have $\sum_{i=0}^{\infty} \hbar^{i} \xi_{\lambda}\left(y_{i}\right)=0$ 
for any $\lambda \in P$. Since $\xi_{\lambda}\left(y_{i}\right) \in k$, we have $\xi_{\lambda}\left(y_{i}\right)=0$ for any $\lambda \in P$, and hence $y_{i}=0$. Therefore we have $\delta(z)=w \cdot \delta(z)$.

4.2. Adjoint Representations. Let $H$ be a (topological) Hopf algebra over $R$. We define an $R$-module homomorphism

by

$$
\text { ad: } H \rightarrow \operatorname{End}_{R}(H)
$$

$$
((\mathrm{ad})(x))(y)=(m \circ(m \otimes 1) \circ \tau)(((1 \otimes S) \Delta(x)) \otimes y),
$$

where $m$ is the product, $S$ is the antipode, $\Delta$ is the coproduct and $\tau: H \otimes H \otimes H \rightarrow$ $H \otimes H \otimes H$ is given by $\tau(a \otimes b \otimes c)=a \otimes c \otimes b$. Then it is easily checked that ad is a homomorphism of $R$-algebras. Therefore the $R$-module $H$ is endowed with an $H$-module structure. We denote $H$ by $H_{\text {ad }}$ when it is regarded as an $H$-module.

We also denote by $\operatorname{rriv}_{H}$ the one-dimensional $H$-module given by the counit $\varepsilon: H \rightarrow R$.

Lemma 4.2.1. (i) For $y \in U_{h}(\mathfrak{g})$, we have $y \in \mathfrak{z}\left(U_{h}(\mathrm{~g})\right)$ if and only if $(\operatorname{ad}(x))(y)=\varepsilon(x) y$ for all $x \in U_{n}(\mathrm{~g})$.

(ii) Let $v$ be a generator of $\operatorname{triv}_{U_{h}(\mathrm{~g})}$. Then we have an isomorphism of $R$-modules

$$
\operatorname{Hom}_{U_{h}(\mathfrak{g})}\left(\operatorname{triv}_{U_{h}(\mathfrak{g})}, U_{h}(\mathfrak{g})_{\mathrm{ad}}\right) \simeq 3\left(U_{h}(\mathrm{~g})\right) \quad(\varphi \leftrightarrow \varphi(v)) .
$$

Proof. The statement (i) is easily verified using (1.17) (1.23), and (ii) is equivalent to (i).

Remark. The definition of the adjoint representation of Hopf algebras and Lemma 4.2.1 are communicated to the author by T. Hayashi.

4.3. Proof of Theorem 2. It remains to show that the algebra homomorphism $\delta: z\left(U_{h}(\mathrm{~g})\right) \rightarrow \bar{T}^{W}$ is an isomorphism. Set

$$
A_{n}=U_{h}(\mathrm{~g}) / \hbar^{n} U_{h}(\mathrm{~g})=U_{h}^{f}(\mathrm{~g}) / \hbar^{n} U_{h^{\prime}}^{f}(\mathrm{~g}), \quad T_{n}=\bar{T} / \hbar^{n} \bar{T}=T / \hbar^{n} T,
$$

for simplicity. As in Sect. 4.1 we have algebra homomorphisms

$$
\delta_{n}: 3\left(A_{n}\right) \rightarrow T_{n}^{W} \quad(n=1,2, \ldots,),
$$

such that the diagram

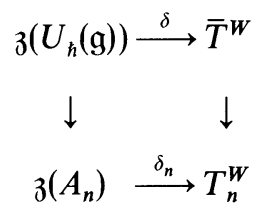

commutes. It is easily seen that we have

$$
\mathfrak{z}\left(U_{\hbar}(\mathfrak{g})\right) \simeq \lim _{\longleftarrow} \mathfrak{z}\left(A_{n}\right), \quad \bar{T}^{W} \simeq \lim _{\leftarrow} T_{n}^{W},
$$

and hence we have only to show that $\delta_{n}: 3\left(A_{n}\right) \rightarrow T_{n}^{W}$ is an isomorphism for each $n$.

Let us prove it by induction on $n$. Since $\delta_{1}$ is the (original) Harish-Chandra isomorphism for $U(\mathrm{~g})$ (see for example [2]), we assume that $n \geqq 2$ and $\delta_{k}$ is an 
isomorphism for $k \leqq n-1$. By Corollary 3.2.1 (i) we have an exact sequence:

$$
0 \rightarrow A_{1} \rightarrow A_{n} \rightarrow A_{n-1} \rightarrow 0,
$$

where $A_{1} \rightarrow A_{n}$ is given by the multiplication by $\hbar^{n-1}$. Hence we have the commutative diagram

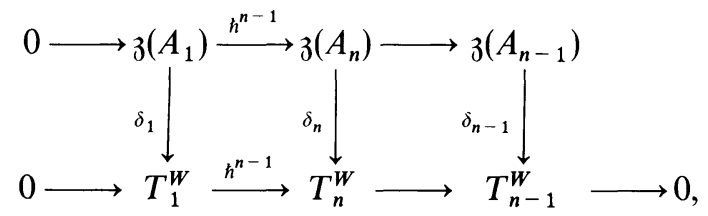

whose rows are exact sequences. Since $\delta_{1}$ and $\delta_{n-1}$ are isomorphisms, it is sufficient to show that $z\left(A_{n}\right) \rightarrow z\left(A_{n-1}\right)$ is surjective.

Similarly to Lemma 4.2 .1 , we have

$$
3\left(A_{n}\right) \simeq \operatorname{Hom}_{A_{n}}\left(\operatorname{triv}_{A_{n}},\left(A_{n}\right)_{\mathrm{ad}}\right)=\operatorname{Hom}_{U_{h}(\mathrm{~g})}\left(\operatorname{triv}_{U_{h}(\mathrm{~g})},\left(A_{n}\right)_{\mathrm{ad}}\right)
$$

and hence it suffices to show

$$
\operatorname{Ext}_{U_{h}(\mathrm{~g})}^{1}\left(\operatorname{triv}_{U_{h}(\mathrm{~g})}, U(\mathrm{~g})_{\mathrm{ad}}\right)=0 .
$$

Let $B \rightarrow C$ be a ring homomorphism. Then for a $B$-module $M$ and a $C$-module $N$ we have the following spectral sequence:

$$
E_{2}^{p, q}=\operatorname{Ext}_{C}^{p}\left(\operatorname{Tor}_{q}^{B}(C, M), N\right) \Rightarrow \operatorname{Ext}_{B}^{p+q}(M, N) .
$$

Let us apply this to the case

$$
B=U_{h}(\mathfrak{g}), \quad C=U(\mathfrak{g}), \quad M=\operatorname{triv}_{U_{h}(\mathfrak{g})}, \quad N=U(\mathfrak{g})_{\mathrm{ad}} .
$$

Using the free resolution:

$$
0 \rightarrow U_{\hbar}(\mathfrak{g}) \stackrel{\hbar}{\rightarrow} U_{\hbar}(\mathfrak{g}) \rightarrow U(\mathfrak{g}) \rightarrow 0 \quad \text { (exact) }
$$

of the right $U_{h}(\mathfrak{g})$-module $U(\mathfrak{g})$, we see that

$$
\operatorname{Tor}_{q}^{U_{h}(\mathrm{~g})}\left(U(\mathfrak{g}), \operatorname{triv}_{U_{h}(\mathrm{~g})}\right)= \begin{cases}\operatorname{triv}_{U(\mathrm{~g})} & q=0 \\ 0 & q \neq 0 .\end{cases}
$$

Hence we have

$$
\operatorname{Ext}_{U_{h}(\mathrm{~g})}^{p}\left(\operatorname{triv}_{U_{h}(\mathrm{~g})}, U(\mathfrak{g})_{\mathrm{ad}}\right)=\operatorname{Ext}_{U(\mathrm{~g})}^{p}\left(\operatorname{triv}_{U(\mathrm{~g})}, U(\mathfrak{g})_{\mathrm{ad}}\right) .
$$

In general we have $\operatorname{Ext}_{U(\mathrm{~g})}^{p}\left(V, \bigoplus_{\lambda} V_{\lambda}\right)=\bigoplus_{\lambda} \operatorname{Ext}_{U(\mathrm{~g})}^{p}\left(V, V_{\lambda}\right)$ for a finite dimensional $U(\mathfrak{g})$-module $V$ and $U(\mathfrak{g})$-modules $V_{\lambda}$. Since $U(\mathfrak{g})_{\text {ad }}$ is a direct sum of finite dimensional $U(\mathfrak{g})$-modules, it is sufficient to show

$$
\operatorname{Ext}_{U(\mathfrak{g})}^{1}\left(\operatorname{triv}_{U(\mathfrak{g})}, V\right)=0
$$

for any finite dimensional $U(\mathfrak{g})$-module $V$. This is a special case of the well-known fact that

$$
\operatorname{Ext}_{U(\mathrm{~g})}^{1}\left(V_{1}, V_{2}\right)=0
$$


for finite dimensional $U(\mathfrak{g})$-modules $V_{1}, V_{2}$. (This is equivalent to the complete reducibility of finite dimensional $U(\mathfrak{g})$-modules.)

The proof of Theorem 2 is completed.

Acknowledgements. The author would like to thank T. Hayashi and H. Yamane for very interesting discussions.

\section{References}

1. Abe, E.: Hopf algebras. Cambridge, New York: Cambridge University Press 1980

2. Dixmier, J.: Algèbres Enveloppantes. Paris: Gauthier-Villars 1974

3. Drinfel'd V. G.: Hopf algebras and the quantum Yang-Baxter equation. Soviet Math. Dokl. 32, 254-258 (1985)

4. Drinfel'd, V. G.: Quantum groups. In: Proc. ICM, Berkeley, 1986. Providence, RI: Am. Math. Soc., 1988

5. Harish-Chandra.: On some applications of the universal enveloping algebra of a semisimple Lie algebra. Trans. Am. Math. Soc. 70, 28-96 (1951)

6. Jimbo, M.: A $q$-difference analogue of $U(\mathfrak{g})$ and the Yang-Baxter equation. Lett. Math. Phys. 10, 63-69 (1985)

7. Jimbo, M.: A $q$-analogue of $U(\mathfrak{g l}(N+1))$, Hecke algebras and the Yang-Baxter equation. Lett. Math. Phys. 11, 247-252 (1986)

8. Kac, V. G.: Infinite dimensional Lie algebras. Progress in Math. Vol. 44, Boston, MA: Birkhäuser 1983

9. Lusztig, G.: Quantum deformations of certain simple modules over enveloping algebras. Adv. Math. 70, 237-249 (1988)

10. Matsumura, H.: Commutative algebra. Second ed. London: Benjamin 1980

11. Rosso, M.: Finite dimensional representations of the quantum analog of a complex simple Lie algebra. Commun. Math. Phys. 117, 581-593 (1988)

12. Serre, J. P.: Algèbres de Lie semi-simples complexes. New York: Benjamin 1966

13. Yamane, H.: A Poincaré-Birkhoff-Witt theorem for quantized universal enveloping algebras of type $A_{N}$, preprint

Communicated by H. Araki

Received June 16, 1989 
